\title{
Rassegna
}

\section{La dieta ipoproteica nella ristorazione collettiva: l'esperienza dell'Azienda Ospedaliero-Universitaria Pisana}

\author{
I. Evangelisti' ${ }^{1}$, C. D’Alessandro² ${ }^{2}$ D. Giannese ${ }^{2}$, E. Colombini ${ }^{2}$ \\ ${ }^{1}$ Servizio Dietetico, U.O. Direzione Medica di Presidio, AUO Pisana, Pisa \\ ${ }^{2}$ Divisione di Nefrologia, Dipartimento di Medicina Interna, Università di Pisa, Pisa
}

Hypoproteic diet in PATIENTS' community: REPORTS From University of Pisa

ABstract. Nutrition is considered by the National Health Authorities as part of the clinical care process. In this perspective, the catering service of a hospital represents a powerful therapeutic and educational aid for the in-patients.

The catering service of our University Hospital in Pisa is based on a collection of standardized diets with indications concerning the type of patient which they are addressed. The present paper deals with our experience in this field, and in particular with the diets for renal patients.

The so called "special" diets, such as low protein $(0.6 \mathrm{~g} / \mathrm{kg} \mathrm{b}$. w. / day) low phosphorus diet, the low protein $(0.7 \mathrm{~g} / \mathrm{kg}$ b.w./ day) vegetarian diet and the very low protein $(0.3 \mathrm{~g} / \mathrm{kg} \mathrm{b}$. w. / day) low phosphorus diet are prescribed by the doctors and developed by the dietician for the individual patient.

Since its preparation, the low-protein diets have several critical points, namely processing - packaging and distribution of the diet, no customization, the low protein artificial foods).

In order to improve the service, we have detected the food actually consumed and the pleasantness of low-protein meals using two survey sheets given to patient during their hospitalization. Overall, the results indicate a good acceptance of diets by patients, but with a reduced consumption of low protein food, bread in particular.

KEY WoRDS. Low-protein diet, In-hospital dietetic intake, Chronic kidney disease, Special dietetic patterns

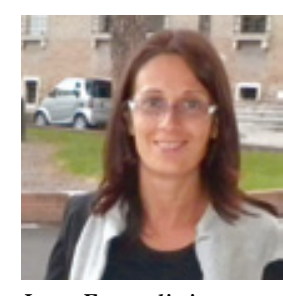

Irene Evangelisti

\section{Introduzione}

La legislazione sanitaria corrente indica come la nutrizione sia da considerare a tutti gli effetti un processo assistenziale, rientrando a far parte integrante della terapia clinica $(1,2)$. Infatti studi scientifici hanno rilevato che attraverso l'integrazione tra dieta e terapia medica si può ottenere un miglioramento dell'efficacia terapeutica, una potenziale diminuzione delle giornate di degenza per singolo paziente e una maggiore stabilità nei risultati clinici raggiunti $(3,4)$. Parallelamente è riconosciuto che un miglioramento dello stato nutrizionale conduce a benefici di natura economica e a un più efficiente utilizzo delle risorse di cura, in termini di riduzione del rischio di ospedalizzazione, ricovero più breve e ridotto rischio di riammissione ospedaliera (5).

In questa ottica il servizio di ristorazione rappresenta un potente ausilio terapeutico in grado di influenzare positivamente o negativamente il decorso ospedaliero $(6,7)$.

\section{Il servizio di ristorazione}

Il servizio di ristorazione dell'Azienda Ospedaliero-Universitaria Pisana prevede un sistema in outsourcing, cioè un appalto esterno totale, sia per il personale che per gli approvvigionamenti.

La cucina centralizzata è ubicata presso la struttura ospedaliera, una per ogni presidio, e si avvale della tecnologia di produzione nota come 'linea calda', che prevede il mantenimento delle pietanze una volta cotte a temperatura non inferiore ai $65^{\circ} \mathrm{C}$ e il trasporto dalla cucina ai reparti con carrelli termici (caldo-freddo).

Il capitolato d'appalto, redatto dalla Direzione Sanitaria in collaborazione col servizio di economato è lo strumento che ne definisce le regole, disciplinando il piano 
organizzativo dell'intero processo. In esso sono specificati tutti gli aspetti del servizio richiesto e i doveri della società di catering appaltatrice (8).

Parte specifica del capitolato è il Dietetico, una raccolta di diete standardizzate a composizione bromatologica definita, che consente di conciliare le esigenze terapeutiche con quelle tecnico-amministrative. Le specifiche indicazioni sulla composizione e la distribuzione dei pasti dettano le basi per il menù ospedaliero, che ha differenziazione semestrale (stagione autunno-inverno e primavera-estate) e articolazione su due settimane. Il Dietetico fornisce la composizione bromatologica delle tipologie di vitto che possono essere richiesti: dal vitto comune, o dieta normocalorica, alle diete speciali standardizzate, ai moduli aggiuntivi (supervitto) per quanto riguarda i degenti e cosi pure per la ristorazione del personale (mensa aziendale).

Le diete standardizzate hanno una composizione definita e sono state create per essere utilizzate nel caso in cui la ditta appaltatrice debba operare una fornitura diretta ai reparti, come ad esempio nei giorni festivi, nel pomeriggio e in caso di chiusura del Servizio Dietetico. Per ognuna di esse sono indicate le finalità e le caratteristiche dei pazienti a cui si rivolge.

Normalmente le diete speciali sono prescritte dal medico, formulate per il singolo paziente, elaborate dal dietista e realizzate utilizzando grammature, alimenti e modalità di preparazione specifiche.

$\mathrm{Nel}$ dietetico ospedaliero ritroviamo la dieta ipofosforica- ipoproteica $(0.6 \mathrm{~g} / \mathrm{kg} / \mathrm{die})$, la dieta ipofosforica-ipoproteica vegetariana $(0.7 \mathrm{~g} / \mathrm{kg} / \mathrm{die})$ e la dieta fortemente ipofosforica ipoproteica $(0.3 \mathrm{~g} / \mathrm{kg} / \mathrm{die})$ supplementata con amminoacidi essenziali e chetoanaloghi, indicate per i pazienti nefropatici (9).

Nelle rispettive composizioni bromatologiche, che rispecchiano gli indirizzi dieto-terapeutici attuali sono indicati il contenuto relativo di protidi, lipidi, glucidi, il contenuto assoluto in colesterolo e fibre, il valore energetico e inoltre il contenuto in proteine animali e vegetali, e dei minerali quali sodio, potassio e fosforo. Il menu ipoproteico ricalca ove possibile quello comune, incorrendo però spesso in modifiche di ricette e di modalità di preparazione dei piatti. Risulta comunque un menu personalizzato variato su due settimane e differenziato su due stagioni, autunno-inverno e primavera-estate. Sono stati previsti, specialmente per i secondi piatti, preparazioni di carne e pesce con pezzatura tale da essere compatibile con le grammature ridotte. La scelta dei piatti viene quindi ad essere mediata dalle necessità terapeutiche, dalle richieste del paziente e dalle esigenze del dietista.

La prescrizione dietetica, di competenza medica, è il punto di partenza dell'intero procedimento.
Il modulo (n. 999-58 codifica aziendale) viene inviato via fax al Servizio Dietetico dell'Azienda Ospedaliera, dove, in base alle informazioni in esso contenute viene elaborata la dieta.

Questa viene poi comunicata al servizio di ristorazione per lo stesso giorno via telefono, altrimenti rientra nella programmazione giornaliera delle diete personalizzate, che vengono consegnate con un giorno di anticipo in modo tale da consentire una migliore organizzazione del servizio.

L'ordine, consistente nella specifica prescrizione del pasto (vassoio personalizzato) e della composizione del sacchetto dei generi di confort (pane, fette biscottate ecc.) viene recepito dal personale addetto alla cucina dietetica. Quest'ultima ha uno spazio ben definito nella cucina centralizzata e ha a disposizione fornelli, frigo, utensili, piano di lavoro, carrelli termici e attrezzature dedicate alla preparazione delle diete speciali.

Attualmente nel presidio di Cisanello, sede delle UU.OO. di Nefrologia e Dialisi, il confezionamento inizia alle ore 9 per il servizio del pranzo e alle ore 15 per quello della cena. L'ordine di confezionamento rispecchia quello di partenza dei carrelli per i reparti.

\section{La preparazione della dieta speciale ipoproteica}

\section{Il vassoio ipoproteico}

L’impiego di prodotti dietetici speciali e la necessità di diversificare le preparazioni e di rispettare in modo rigoroso le grammature dei secondi piatti hanno portato all'elaborazione di procedure e istruzioni operative, che vengono continuamente revisionate al fine di migliorare sempre di più il servizio.

\section{Primi piatti}

Pasta aproteica: la ridotta tenuta in cottura determinata dalla privazione delle proteine (glutine) comporta una procedura di lavorazione totalmente diversa, frutto di numerose prove di cottura-resa-qualità. Attualmente la pasta viene cotta all' $80 \%$ (molto al dente) del tempo di cottura dichiarato dalla ditta produttrice, con alcune differenze in base al formato stesso, poi viene raffreddata con acqua fredda e fermata con olio extravergine di oli$\mathrm{va}$, in seguito condita, sporzionata e messa col vassoio nel carrello 'Burlodge', dove viene rigenerata e mantenuta al caldo $\left(75^{\circ} \mathrm{C}\right)$ fino al momento del consumo. $\mathrm{Al}$ contatto con la salsa e per mezzo del calore del carrello la pasta riprende a cuocere. Ciò ha comportato la necessità di fare due cotture distinte per ogni servizio, al fine di ridurre il tempo intercorrente tra preparazione e con- 
sumo, per ottenere benefici sulla qualità (palatabilità) del piatto. Lo sporzionamento si basa sul peso a cotto del prodotto e tiene conto delle variazioni di peso che la pasta subisce durante la cottura (resa in cottura 140\%) e del peso della salsa di condimento (circa $30-40 \mathrm{~g}$ a porzione). Riso: viene cotto per 30 minuti circa in abbondante acqua, condito e poi sporzionato secondo la resa a cotto $(+300 \%)$.

Pasta di semola: viene cotta in abbondante acqua senza sale per il tempo indicato nella confezione, scolata, addizionata della salsa e poi sporzionata secondo grammatura tenendo conto della resa a cotto.

\section{Secondi piatti}

Anche nel porzionamento dei secondi piatti viene presa in considerazione la resa a cotto del prodotto. Con varie prove di cottura, che hanno preso in considerazione materie prime diverse, è stato rilevato il calo reale del prodotto ed elaborata una tabella di conversione dei pesi da crudo a cotto. Questo procedimento viene applicato per tutte le preparazioni elaborate (arrosti, bolliti...) in cui la cottura non può avvenire in monoporzione. Le preparazioni semplici, quali fettine, scaloppine e pesce vengono preparate secondo la grammatura a crudo direttamente dal reparto macelleria. I legumi secchi e di origine biologica, per le diete vegane, vengono messi in ammollo la sera prima, scolati e lavati in acqua corrente e poi lessati in acqua. La preparazione spesso è per monoporzione, visto che le diete vegane non sono molto frequenti.

Gli albumi d'uovo utilizzati per preparare omelette o frittate con verdura, vengono proposti una volta a settimana. Il prosciutto cotto, presente in menu una volta a settimana, è privo di polifosfati aggiunti e di glutine.

\section{Contorni}

Le verdure vengono cotte in forno a vapore ed eventualmente saltate in brasiera con olio extravergine di oliva.

\section{Il sacchetto ipoproteico}

I sacchetti con i generi di comfort di accompagnamento (secchi) della dieta vengono preparati in una zona di confezionamento dedicata della cucina. A seconda della prescrizione possiamo ritrovare:

- Marmellata in monoporzione

- Burro

- Zucchero in bustine

- Olio extravergine di oliva in bustine monodose

- Pane senza sale

- Frutta fresca di stagione
Prodotti a fini medici speciali quali:

- Pancarrè aproteico

- Fette tostate aproteiche

- Biscotti aproteici

Il sacchetto viene posto sul vassoio del pranzo e contiene i generi di comfort per l'intera giornata.

\section{I punti critici nel percorso della dieta ipoproteica}

\section{Elaborazione dieta}

La prescrizione dietetica risulta essere un punto critico, poiché talvolta è incompleta di alcuni dei dati richiesti nel modulo (antropometrici, in primis) e non viene indicata la patologia di interesse dietetico ma quella motivo di ricovero. Ciò comporta un ulteriore contatto telefonico del dietista con il reparto.

Per ovviare alla mancata personalizzazione della dieta, vista l'impossibilità del Servizio Dietetico di rilevare personalmente i gusti e le abitudini alimentari di tutti pazienti a dieta, sono state elaborate due schede (A-B) da far compilare al momento del ricovero e inviare via fax al servizio dietetico.

- Scheda A: consente la rilevazione dei consumi abituali. E costituita da 2 parti, la prima indaga la tipologia e distribuzione giornaliera dei prodotti dietetici e degli alimenti consumati abitualmente. Nella seconda vengono registrati gli alimenti e le preparazioni presenti nel menu che non sono gradite al paziente. Tale scheda permette di personalizzare la dieta tenendo conto di gusti e abitudini.

- Scheda B: ha lo scopo di monitorare i consumi effettivi dei pasti ipoproteici. Rileva la percentuale del pasto consumato e consente di esprimere un giudizio sulla qualità del pasto servito. Tale scheda ci permette di evidenziare eventuali problematiche nelle preparazioni dei piatti e allo stesso tempo di monitorizzare la compliance alla terapia dietetica.

\section{Confezionamento dieta}

Le procedure attualmente messe in atto per la preparazione delle diete ipoproteiche hanno consentito di ridurre il problema della scarsa tenuta in cottura e del rispetto delle grammature del secondo piatto.

Per quanto riguarda i contorni, la cottura a vapore delle verdure non garantisce la perdita ottimale in micronutrienti, ma attualmente non sono state valutate soluzioni alternative.

Punto critico rimane l'utilizzo del pane aproteico in 

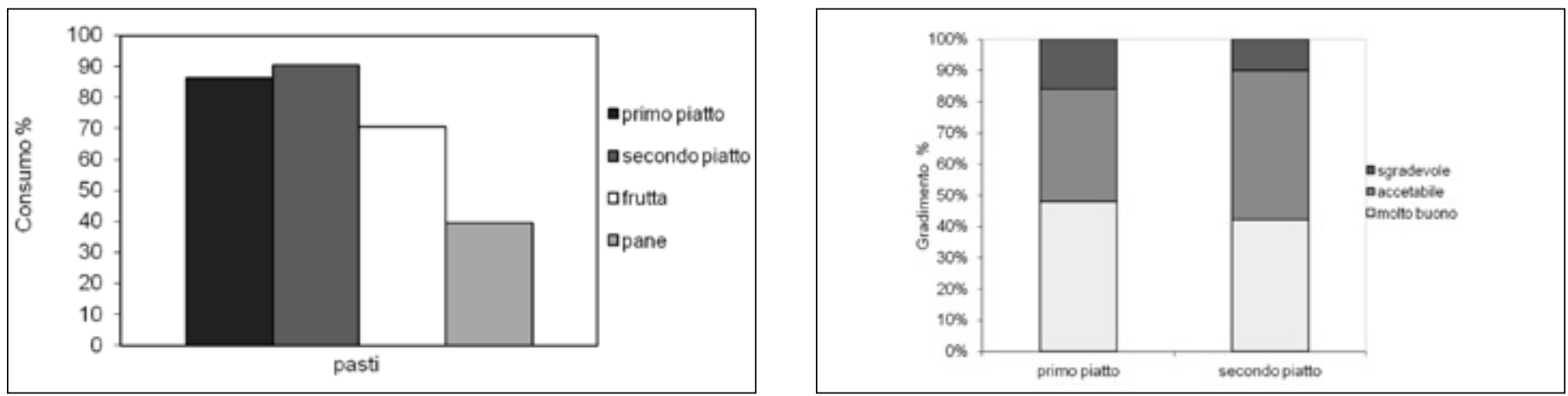

Fig. 1 - Risultati relativi al consumo e alla gradevolezza (espressi in percentuale) della dieta ipoproteica $(0.6 \mathrm{~g} / \mathrm{kg} /$ die) ipofosforica standard.
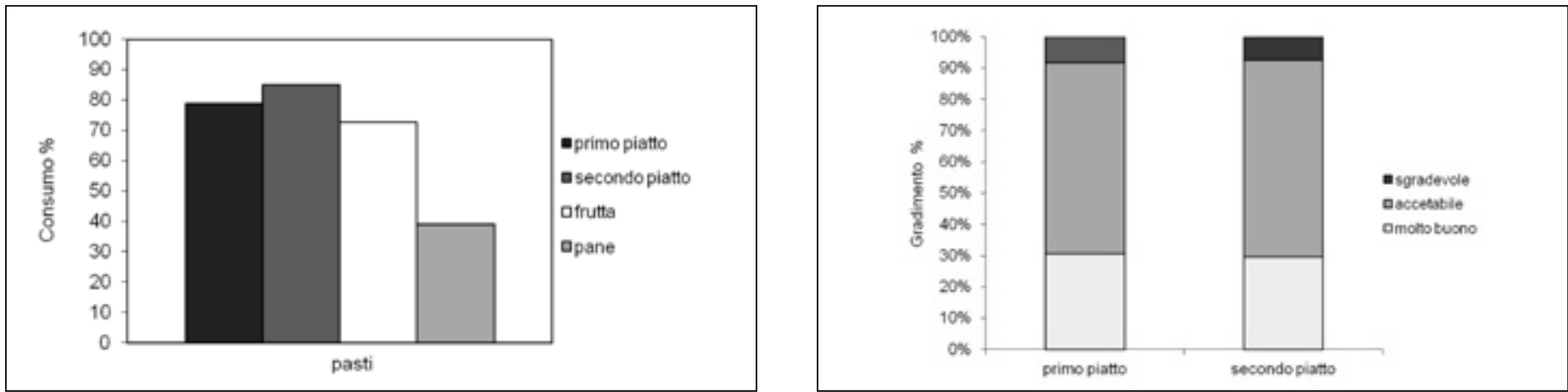

Fig. 2 - Risultati relativi al consumo e alla gradevolezza (espressi in percentuale) della dieta ipoproteica $(0.7 \mathrm{~g} / \mathrm{kg} /$ die) ipofosforica vegetariana.
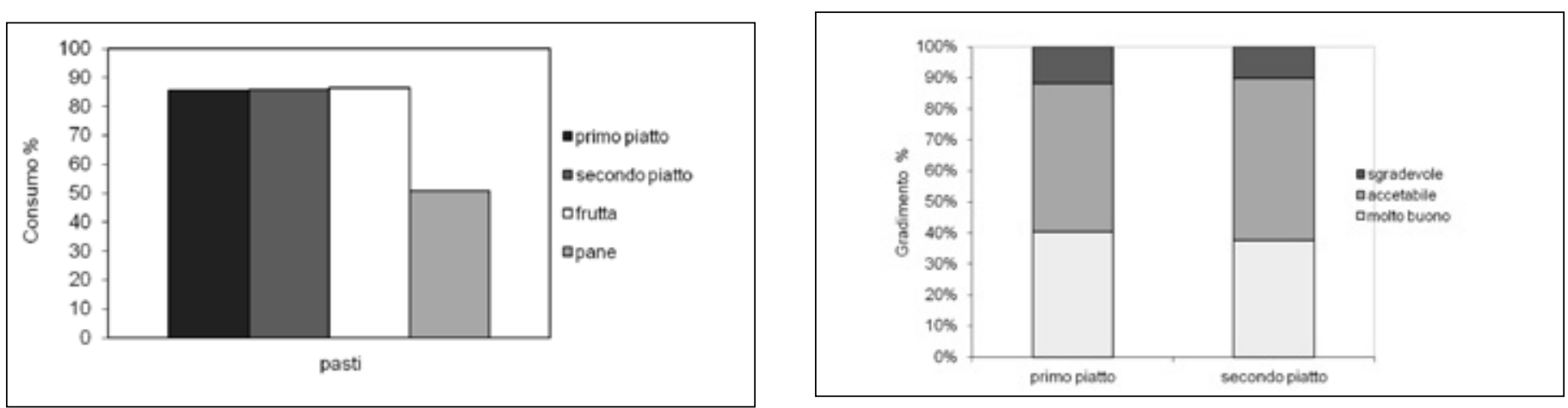

Fig. 3 - Risultati relativi al consumo e alla gradevolezza (espressi in percentuale) della dieta fortemente ipoproteica $(0.3 \mathrm{~g} / \mathrm{kg} / \mathrm{die})$ ipofosforica supplementata con aminoacidi essenziali e chetoanaloghi.

formato pancarrè, che, non potendo essere riscaldato prima del consumo, risulta poco gradito. Per garantire comunque un sufficiente apporto calorico, pur limitando il consumo del pane, vengono fornite porzioni di pasta abbondanti sia a pranzo che a cena, e nel sacchetto vengono distribuite fette tostate e biscotti ipoproteici in grammatura superiore alle reali necessità.
Distribuzione vitto in corsia

Gli eventuali errori nella distribuzione del vitto, per attribuzione dei vassoi a pazienti differenti, si sono ridotti da quando il vassoio con dieta speciale viene presentato con stoviglie monouso, e accompagnato dal biglietto indicante reparto, numero di letto e cognome del paziente. Sul biglietto compare inoltre la specifica composizione del pasto presentato, comprensivo di grammatura, in 
modo tale che anche il paziente può controllare la corrispondenza della sua dieta.

Nel processo che porta alla distribuzione del pasto - dieta speciale - al paziente ricoverato intervengono, quindi, diversi attori, tutti con ruoli e con responsabilità specifiche.

Per garantire il soddisfacimento delle necessità e delle aspettative dietetiche nutrizionali di ciascun degente è fondamentale la mobilitazione e il coinvolgimento di tutto il sistema operativo.

Il Servizio Dietetico rappresenta in quest'ottica l'anello di congiunzione tra il processo assistenziale ospedaliero e il servizio di ristorazione, grazie alla specificità professionale del Dietista, riportata nelle posizioni ANDID, «...di saper coniugare gli aspetti organizzativi e igienico sanitari a quelli nutrizionali»(10).

\section{La nostra esperienza}

Allo scopo di verificare la gradevolezza e il consumo dei pasti speciali distribuiti durante la degenza, abbiamo analizzato le schede 'B' di 25 pazienti (20 maschi, 5 femmine) ricoverati presso la U.O. Nefrologia 1 Universitaria in Pisa, per un totale di 205 pasti (106 pranzi e 99 cene). I pazienti avevano un'età compresa tra 20 e 75 anni ed erano tutti affetti da insufficienza renale cronica (III-V stadio NKF).

Di questi, 16 pazienti seguivano la dieta ipoproteica (0.6 $\mathrm{g} / \mathrm{kg} / \mathrm{die}$ )-ipofosforica standard, 5 la dieta ipoproteica $(0.7 \mathrm{~g} / \mathrm{kg} / \mathrm{die})$-ipofosforica vegana, e 4 la dieta fortemente ipoproteica $(0.3 \mathrm{~g} / \mathrm{kg} /$ die)-ipofosforica supplementata con amminoacidi essenziali e chetoanaloghi.

Le Figure 1-3 mostrano i dati relativi al consumo e alla gradevolezza (espressi entrambi in percentuale) rispettivamente della dieta ipoproteica $(0.6 \mathrm{~g} / \mathrm{kg} / \mathrm{die})$ ipofosforica standard, della dieta ipoproteica $(0.7 \mathrm{~g} / \mathrm{kg} / \mathrm{die})$ ipofosforica vegetariana e della dieta fortemente ipoproteica $(0.3 \mathrm{~g} / \mathrm{kg} / \mathrm{die})$ ipofosforica supplementata con amminoacidi essenziali e chetoanaloghi.

Il consumo e la gradevolezza si riferiscono ad entrambi i pasti; la gradevolezza è riferita soltanto al primo e al secondo piatto, non al pane e alla frutta. Dai dati osserviamo che la percentuale di consumo del primo piatto (costituito dalla pasta aproteica) da parte dei pazienti che seguono la dieta ipoproteica $0.6 \mathrm{~g} / \mathrm{kg} /$ die e quella $0.3 \mathrm{~g} /$ $\mathrm{kg} /$ die è di circa l'80\%; la stessa percentuale si osserva nei pazienti che seguono la dieta vegetariana, dove il primo piatto è costituito da pasta di semola di grano duro. Inoltre l'analisi dei dati relativi alla gradevolezza, a parità di percentuale di consumo, la pasta aproteica risulta avere un indice di gradimento maggiore rispetto alla pasta di semola di grano duro. Questo potrebbe essere dovuto alle diverse aspettative che il paziente ha verso il prodotto aproteico (che, in quanto diverso, viene considerato meno buono a priori) e/o alla maggiore attenzione che viene posta durante la preparazione alla cottura della pasta aproteica rispetto a quella comune.

Appare eclatante il dato relativo al consumo del pane che risulta essere inferiore al 50\% per tutti e tre i tipi di dieta. Questo potrebbe essere dovuto alla scarsa palatabilità del pancarrè aproteico, per le diete che prevedono l'utilizzo degli alimenti aproteici e alla consuetudine di non consumare il pane senza sale e/o di accompagnarlo al secondo piatto 'proteico', per la dieta vegetariana ipoproteica.

Esiste quindi la necessità di integrare le calorie mancanti sottoforma di altre fonti di carboidrati.

Sulla base di questi risultati, la cucina della nostra AOUP di Pisa ha iniziato ad inviare porzioni più abbondanti, con un aumento del primo piatto sia a pranzo che a cena, distribuzione nel sacchetto di fette tostate e biscotti ipoproteici in grammatura superiore alle reali necessità, in modo da realizzare vassoi che hanno un apporto calorico del $20 \%$ superiore rispetto a quello prescritto.

In conclusione, nonostante la complessità e le difficoltà che si hanno con l'alimentazione ipoproteica nella ristorazione ospedaliera, i nostri risultati indicano una buona accettazione delle diete da parte dei pazienti, anche se si registra un ridotto consumo in particolare riguardante il pane aproteico.

\section{Riassunto}

La nutrizione è considerata dalla legislazione sanitaria corrente parte integrante della prevenzione e terapia clinica, e quindi del processo assistenziale. In questa ottica il servizio di ristorazione di un ospedale rappresenta un potente ausilio terapeutico ed educazionale.

Il servizio di ristorazione della nostra Azienda Ospedaliera-Universitaria Pisana si basa sul Dietetico, una raccolta di diete standardizzate a composizione bromatologica definita, nel quale vengono indicate anche le finalità e le caratteristiche dei pazienti a cui si rivolge. Questo articolo riporta la nostra esperienza in questo settore, con particolare riguardo alle diete speciali per i pazienti nefropatici.

Le diete speciali, come la dieta ipoproteica $(0.6 \mathrm{~g} / \mathrm{kg}$ p.c./die) ipofosforica, la dieta ipoproteica $(0.7 \mathrm{~g} / \mathrm{kg}$ p.c./ die) vegetariana e la dieta fortemente ipoproteica $(0.3$ $\mathrm{g} / \mathrm{kg} \mathrm{p.c./die)} \mathrm{ipofosforica} \mathrm{sono} \mathrm{prescritte} \mathrm{dal} \mathrm{medico,}$ elaborate dal dietista e formulate per il singolo paziente. Sin dalla sua preparazione la dieta speciale ipoproteica presenta diversi punti critici (elaborazione-confezionamento e distribuzione della dieta, mancata personalizzazione, prodotti artificiali aproteici). Allo scopo di mi- 
gliorare il servizio dietetico abbiamo rilevato l'effettivo consumo e la gradevolezza dei pasti speciali ipoproteici distribuiti durante la degenza mediante la compilazione di due schede consegnate ai pazienti nel corso della degenza. Nel complesso i risultati indicano una buona accettazione delle diete da parte dei pazienti, ma con un ridotto consumo in particolare riguardante il pane aproteico.

Parole Chiave. Dieta ipoproteica, Dieta ospedaliera, Insufficienza renale cronica, Dieta speciale

IL PRESENTE ELABORATO È STATO PRESENTATO DALL'AUTORE, QUALE pRova finale, al Master DI PRIMO LIVELLO "NUTRIZIONE E DIeteTica IN NEFrologia” DELL'UNIVERSITÀ DI PISA.

\section{Indirizzo dell'Autore:}

Dott.ssa Irene Evangelisti, Dietista

Servizio Dietetico

U.O. Direzione Medica di Presidio

AOU Pisana, Ospedale di Cisanello

via Paradisa 2

56126 Pisa

ireneevang@libero.it

Il presente elaborato è stato presentato dall'Autore, quale prova finale, al Master di primo livello "Nutrizione e Dietetica in Nefrologia” dell'Università di Pisa

\section{Bibliografia}

1. Ministero della salute. Rapporti ISTISAN n. 42 anno 2009.

2. Council of Europe. Public Health Committee. Committee of expert on nutrition, food safety and consumer health. Ad hoc group nutrition programmes in hospitals. Food and nutritional care in hospitals: how to prevent undernutrition. Reports and Guidelines Strasbourg 2002.

3. Comi D, Palmo A, Brugnani M, et al. The hospital malnutrition Italian study. Clin Nutr 1998;17(Suppl): S52.

4. Kondrup J. Proper hospital nutrition as a human right. Clin Nutr 2004; 23(2): 135-7.

5. Cereda E, Lucchin L, Pedrolli C, D’Amicis A, Gentile MG, Battistini NC, Fusco MA, Palmo A, Muscaritoli M. Nutritional care routines in Italy: results from the PIMAI (Project: Iatrogenic MAlnutrition in Italy) study. Eur J Clin Nutr 2010; 64(8): 894-8.

6. Pedrolli C, Costa A. Ristorazione Ospedaliera. In: Dietetica e Nutrizione, Clinica, terapia e organizzazione, G. Fatati, Il Pensiero Scientifico Editore 2007; 731-42.

7. Ferrari P, Iacono G. Manuale giuridico-tecnico per la ristorazione collettiva. Maggioli Editore.

8. Ministero della salute. Linee di indirizzo nazionale per la ristorazione ospedaliera e assistenziale. Settembre 2010.

9. Cupisti A. Definizione della terapia nutrizionale nel trattamento conservativo della IRC. 2008 Sep-Oct; 25(Suppl) 42: S35-8.

10. Agostini S, Cecchetto G, Masini ML, Troiano E, Vezzosi S. Ruolo del Dietista nella prevenzione e nel trattamento della malnutrizione per difetto nel paziente ospedalizzato e/o istituzionalizzato. In Il Dietista e la pratica professionale: Posizioni dell'ANDID. Associazione Nazionale Dietisti; 2004: 22-25. 